\title{
DINÂMICA DE SISTEMAS APLICADA À GESTÃO DE RECURSOS HÍDRICOS EM BACIA HIDROGRÁFICA ${ }^{1}$
}

Wilson da Cruz Vieira ${ }^{2}$

\begin{abstract}
Resumo: Este trabalho objetivou analisar a alocação de recursos hídricos entre demandas competitivas em bacias hidrográficas e políticas de preços para a água. Com base em um modelo teórico que maximiza os benefícios sociais líquidos da alocação de água ao longo de um período finito de tempo, utilizou-se a abordagem de dinâmica de sistemas na análise de uma série de questões relacionadas com gestão de recursos hídricos em bacias hidrográficas, tais como sustentabilidade no uso desses recursos e cobrança pelo uso da água. O modelo foi aplicado à bacia hidrográfica do rio Araguari, localizada no Estado de Minas Gerais.
\end{abstract}

Palavras-chave: bacia hidrográfica, dinâmica de sistemas, recursos hídricos, preço da água, sustentabilidade.

\section{Introdução}

Por ser um recurso renovável e existir em abundância na natureza, há a falsa impressão de que a água é um recurso inesgotável. Entretanto, 97\% da água do planeta encontra-se nos oceanos e não pode ser usada diretamente para irrigação, uso doméstico e, ou, uso industrial. Por um lado, dos $3 \%$ restantes, grande parte $(2 \%)$ está na forma de geleiras e calotas polares de difícil acesso e apenas $1 \%$ do total da água doce está disponível e pode ser usada diretamente pelo homem (TUNDISI, 2003); por outro, a distribuição dos recursos hídricos é bastante desigual entre países e mesmo entre regiões de um país, o que, associado aos problemas ambientais e ao desperdício no seu uso, tem levado ao aumento de sua escassez relativa.

Recebido em 15/09/2008; Aceito em 10/12/2008.

Professor Associado do Departamento de Economia Rural, Universidade Federal de Viçosa. CEP 36570-000 Viçosa - MG. E-mail: wvieira@ufv.br. 
No Brasil, os recursos hídricos são abundantes, mas mal distribuídos. O país detém cerca de $16 \%$ do total das reservas mundiais de água doce utilizável, mas $68,5 \%$ de suas reservas encontram-se na região Norte, que detém apenas $7 \%$ da população. Na região Sudeste, com $42,6 \%$ da população do país, a água doce disponível corresponde a apenas $6 \%$ (REVISTA SUL AMBIENTAL, 2003). Em razão dessa distribuição irregular da água doce no país, alguns problemas relativos a esse recurso têm-se manifestado com maior intensidade nas regiões com baixa relação recursos hídricos/população, sendo as regiões Nordeste e Sudeste as mais críticas.

Com o aumento do consumo de água, decorrente dos crescimentos da população e das atividades econômicas, têm surgido diversos tipos de conflitos relacionados não só com sua utilização, mas também com sua gestão. Leal (1998) citou três desses conflitos: a) Entre usuários ocorrem devido às limitações na disponibilidade de água em quantidade e, ou, na qualidade necessária para atender aos diversos usuários; b) Intersetoriais - surgem entre diferentes usos possíveis na disputa quanto às prioridades estabelecidas em planos de desenvolvimento; e c) Regionais - ocorrem quando a bacia hidrográfica é formada por vários estados, entre municípios a montante e a jusante e entre bairros e comunidades.

Essas várias possibilidades de conflitos relacionados com uso e gestão das águas e suas fortes características de bem público têm levado o setor público, de diversos países, a regularem e criarem instrumentos para gerir os recursos hídricos. No Brasil, o marco legal das questões hídricas está definido pelo Código Civil de 1916, pelo Código das Águas de 1934, ainda em vigor, pelas Leis Federais No. 6.938/81 e No. 9.433/ 97, e pelas leis e decretos estaduais ${ }^{3}$. O Código das Águas assegura o uso das águas para as primeiras necessidades da vida e prevê, entre outras coisas, concessão ou autorização para uso de águas por

Textos das legislações federais e estaduais sobre recursos hídricos podem ser obtidos no endereço eletrônico: http://www.abrh.org.br (2007). 
determinado período de tempo (não superior a trinta anos) e uso comum de águas públicas na forma gratuita ou remunerada.

A atual política nacional de recursos hídricos, definida pela Lei Federal No. 9.433, de 8 de janeiro de 1997, baseia-se nos seguintes fundamentos: ${ }^{4}$ a) A água é um bem de domínio público; b) É um recurso natural limitado, dotado de valor econômico; c) Em situações de escassez, o uso prioritário dos recursos hídricos é para consumo humano e para saciar a sede de animais; d) A gestão dos recursos hídricos deve sempre proporcionar o uso múltiplo das águas; e) A bacia hidrográfica é a unidade territorial para implementação da política nacional de recursos hídricos; e f) A gestão dos recursos hídricos deve ser descentralizada e contar com a participação do Poder Público, dos usuários e das comunidades.

Dentre os aspectos institucionais mais importantes da atual Política Nacional de Recursos Hídricos, destacam-se: a) A bacia hidrográfica é a unidade territorial para implementação da Política Nacional de Recursos Hídricos; e b) A gestão dos recursos hídricos deve ser descentralizada e contar com a participação do Poder Público, dos usuários e das comunidades. Nesse sentido, a Lei Federal No. 9.433/97 prevê a criação de Comitês de Bacia Hidrográfica cujas áreas de atuação incluem: a) A totalidade de uma bacia hidrográfica; b) Sub-bacia hidrográfica de tributário do curso de água principal da bacia, ou de tributário desse tributário; ou c) Grupo de bacias ou sub-bacias hidrográficas contíguas.

Compete aos Comitês de Bacia Hidrográfica, no âmbito de sua área de atuação, entre outras coisas: a) Promover o debate das questões relacionadas com recursos hídricos e articular a atuação das entidades intervenientes; b) Arbitrar, em primeira instância administrativa, os conflitos relacionados com recursos hídricos; c) Aprovar o Plano de Recursos Hídricos da bacia e acompanhar sua execução; d) Estabelecer os mecanismos de cobrança pelo uso de recursos hídricos e sugerir os valores a serem cobrados; e e) Estabelecer critérios e promover o rateio de custo das obras de uso múltiplo, de interesse comum ou coletivo.

4 Esses fundamentos constam no Título I, Capítulo I, Art. 1º., da Lei Federal No. 9.433, de 8 de janeiro de 1997. 
Dada a importância desse novo arranjo institucional e legal para a gestão de recursos hídricos no Brasil, este trabalho objetivou modelar uma bacia hidrográfica (bacia do rio Araguari, localizada no Estado de Minas Gerais), tendo em vista o planejamento e a gestão dos recursos hídricos desta bacia, em conformidade com a legislação brasileira. De forma específica, buscou-se modelar a disponibilidade física de água (superficial e subterrânea) desta bacia e sua inter-relação com os diversos usuários (consumos urbano, industrial e agrícola) e analisar cenários alternativos de gestão dos recursos hídricos, tais como sustentabilidade no uso desses recursos e efeitos econômicos da cobrança pelo uso da água.

\section{Caracterização da bacia do rio Araguari}

A bacia do rio Araguari está localizada na porção oeste do Estado de Minas Gerais (conhecida como Triângulo/Alto Paranaíba), região Sudeste do Brasil, e cobre uma área de $20.186 \mathrm{~km}^{2}$ (ver Figura 1). Esta área está dividida entre 20 municípios (Araguari Araxá, Campos Altos, Ibiá, Indianápolis, Iraí de Minas, Nova Ponte, Patrocínio, Pedrinópolis, Perdizes, Pratinha, Rio Paranaíba, Sacramento, Santa Juliana, São Roque de Minas, Serra do Salitre, Tapira, Tupaciguara, Uberaba e Uberlândia), a maioria dos quais (treze) possui apenas parte de sua área na bacia. Em 2007, a população desses municípios somava 1,4 milhão (IBGE, 2008). 


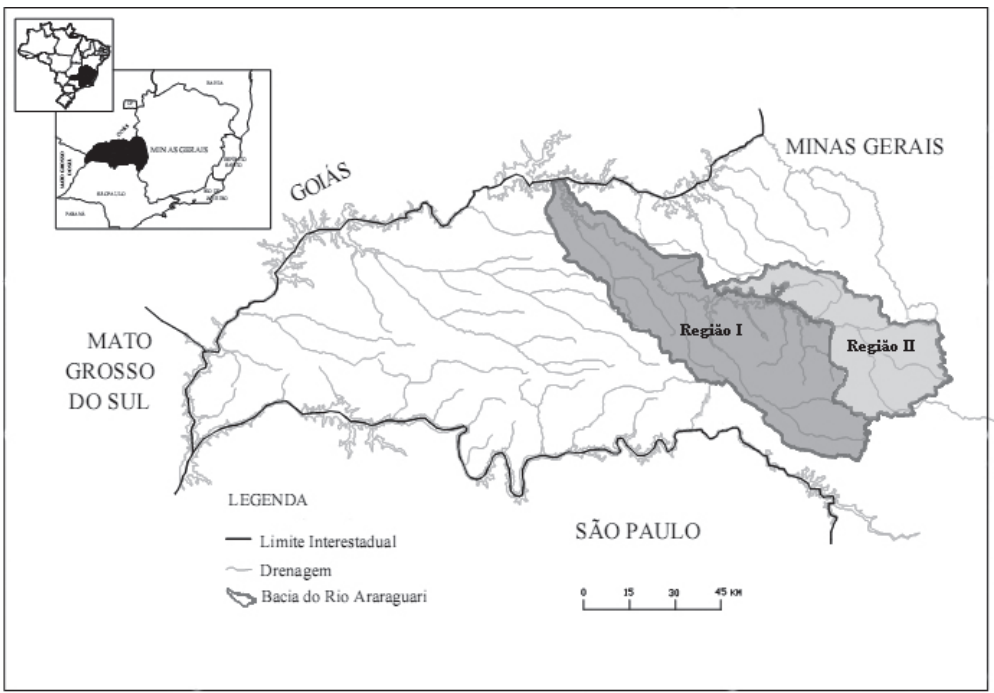

Figura 1 - Localização geográfica da bacia do rio Araguari.

O Instituto Mineiro de Gestão das Águas (IGAM) administra as outorgas de água no Estado de Minas Gerais; analisa solicitações de novas outorgas, assim como pedidos de renovações de outorgas; e as aprova ou não, de acordo com as Lei Federal No. 9.433/97 e Lei Estadual No. 11.504/94. Usos insignificantes de água não estão sujeitos a outorgas, tais como água para satisfazer às necessidades de pequenos grupos populacionais distribuídos em áreas rurais. No Estado de Minas Gerais, as outorgas são divididas basicamente em três tipos: a) As de utilidade pública e usualmente válidas por 20 anos; b) As que não são de utilidade pública e cujos níveis de captação de água não são considerados pequenos e usualmente válidas por cinco anos; e c) As que não são de utilidade pública e cujos níveis de captação de água são considerados pequenos e usualmente válidas por três anos. 
Cada outorga de água para dado usuário indica o município, o local de captação (latitude e longitude), a fonte de água (superficial ou subterrânea), o propósito (consumo humano, irrigação ou uso industrial, por exemplo), o nível de captação em $\mathrm{m}^{3}$ por hora, o número de horas de captação por dia e o número de meses de captação por ano. Com essas informações, pode-se calcular, com razoável precisão, o nível de consumo de água em cada município de dada bacia. Para implementar o modelo desenvolvido na próxima seção, a bacia do rio Araguari foi dividida em duas regiões (ver Figura 1) - a região I, composta pelos municípios Araguari, Araxá, Indianápolis, Nova Ponte, Pedrinópolis, Perdizes, Sacramento, Santa Juliana, São Roque de Minas, Tapira, Tupaciguara, Uberaba e Uberlândia, e a região II, pelos municípios Campos Altos, Ibiá, Iraí de Minas, Patrocínio, Pratinha, Rio Paranaíba e Serra do Salitre. A Tabela 1 apresenta os níveis de consumo de água nessas duas regiões em 2006, os quais foram ajustados de acordo com a área de cada município na bacia.

Tabela 1 - Consumos de águas superficial e subterrânea na bacia do rio Araguari em mil m , 2006, Estado de Minas Gerais

\begin{tabular}{lrrrrr}
\hline Região & \multicolumn{5}{c}{ Água Superficial } \\
\hline \multicolumn{7}{c}{ Urbano } & \multicolumn{1}{c}{ Agrícola } & Industrial & \multicolumn{1}{c}{ Total } & Percentagem \\
\hline Região I & $132.412,25$ & $155.072,63$ & $38.099,50$ & $325.584,38$ & 19,09 \\
Região II & $21.751,50$ & $1.355 .821,73$ & $2.770,28$ & $1.380 .343,50$ & 80,91 \\
Total & $154.163,74$ & $1.510 .894,36$ & $40.869,78$ & $1.705 .927,88$ & 100,00 \\
\hline \multicolumn{5}{c}{ Água Subterrânea } \\
\hline \multicolumn{7}{c}{ Região I } & Urbano & Irrigação & Industrial & Total & Percentagem \\
\hline Região II & $20.465,10$ & $53.834,48$ & $11.151,13$ & $85.450,72$ & 97,45 \\
Total & $1.478,72$ & 467,43 & 293,96 & $2.240,12$ & 2,55 \\
\hline
\end{tabular}

Fonte: IGAM (2008).

Em 2006, o consumo de água na bacia do rio Araguari foi de 1.793.618,72 mil $\mathrm{m}^{3}$, dividido entre as duas fontes de água da seguinte forma: água superficial $(95,11 \%)$ e água subterrânea $(4,89 \%)$. Como pode ser observado na Tabela 1 , o consumo de água na região I é basicamente de água superficial $(80,91 \%)$ e na região II, de água subterrânea $(97,45 \%)$. Em 2006, o consumo de água por setor estava dividido da seguinte forma: a) Água superficial: urbano $(9,04 \%)$, agrícola $(88,57 \%)$ e industrial 
(2,40\%); e b) Água subterrânea: urbano (25,02\%), agrícola (61,92\%) e industrial $(13,05 \%)$. Esses dados mostram que o setor agrícola (irrigação) constitui o principal consumidor de água na bacia do rio Araguari. As principais culturas irrigadas incluem café, milho, feijão e pastagem.

\section{Modelo teórico}

Para os propósitos desde trabalho, o mercado competitivo foi usado como benchmark na alocação de água, dado que no Brasil as outorgas de água não são comercializáveis. O modelo considera a alocação de água renovável escassa em um ambiente dinâmico. A oferta disponível de água vem de duas fontes distintas: água superficial e água subterrânea. Assume-se que as ofertas de águas para captação em uma bacia hidrográfica venham de água superficial (água em rios, reservatórios e fluxos na superfície do solo) e de água subterrânea (água renovável, isto é, a fração de água que infiltra no solo e se acumula em aquíferos). Água para uso não-consuntivo (tais como água para navegação, turismo ou serviços ambientais) não foi considerada explicitamente no modelo. Os usos de água nesta bacia encontram-se sob regulação, e o gestor de recursos hídricos (comitê de bacia/instituição responsável pelas outorgas) deve estabelecer, entre outras coisas, quanto cobrar pelo uso da água.

Tendo em vista uma melhor gestão dos recursos hídricos, uma bacia hidrográfica pode ser dividida em várias regiões, $i=1,2, \ldots, N$, em que $i$ representa a $i$-ésima região. Em cada região pode existir um ou mais setores que demandam água, $j=1,2, \ldots, M$, em que $j$ representa o $j$ ésimo setor. Utilizam-se $S$ e $G$ para representar água superficial e água subterrânea, respectivamente. Assim, $S_{i j}(t)=s(P(t))$ é a demanda de água superficial na região $i$ e setor $j$, no período $t ; G_{i j}(t)=g(P(t))$ é a demanda de água subterrânea na região $i$ e setor $j$, no período $t$; e $P(t)$, preço da água no período $t$. 
Com base nessas definições iniciais, pode-se definir a demanda agregada de água em cada região. Assim, as demandas de águas superficial e subterrânea da região $i$, no período $t$, são representadas, respectivamente, por $S_{i}(t)=\sum_{j} S_{j}(t)$ e $G_{i}(t)=\sum_{j} G_{j}(t)$. As demandas agregadas de águas superficial e subterrânea em toda a bacia, no período $t$, são representadas, respectivamente, por $S(t)=S(P(t))$, em que $S(t)=\sum_{i} \sum_{j} S_{i j}(t)$, e $G(t)=G(P(t))$, em que $G(t)=\sum_{i} \sum_{j} G_{i j}(t)$. A demanda agregada inversa de água em toda a bacia é representada pela função $P(t)=D(W(t))$, em que $W(t)=G(t)+S(t)$. Assumiuse que todas essas funções de demanda sejam diferenciáveis e possuam inclinação negativa.

Para completar as suposições básicas do modelo, assumiu-se também que: a) Os custos externos (poluição da água, por exemplo) estão associados apenas ao uso de água superficial, são considerados constantes na bacia e representados por $E$; b) Os custos marginais de captação de água superficial são constantes na bacia e representados por $C$; c) Os custos marginais de captação de água subterrânea são constantes na bacia e representados por $K$; d) $K$ é igual ou estritamente maior do que $C$; e) Os usuários de água de cada setor e de cada região obtêm outorgas para uso desse recurso na bacia por um período limitado de tempo $[0, T]$. Por simplicidade, assumiu-se que a reposição de águas superficial e subterrânea depende do estoque de água na bacia.

A função de oferta de água na bacia, representada por $C M$, tem o formato indicado na Figura 2 (quando $K>C$ ) e apresenta as seguintes propriedades (a variável $t$ foi suprimida): $C M(W)=C$ para $0<W<S$ e $C M(W)=K$ para $S<W .^{5}$ A curva de demanda agregada de água na bacia é representada por $D$ e consiste da soma horizontal das curvas de

Para uma construção similar de uma curva de oferta, ver Brill et al. (1997). 
demandas agregadas de água superficial e água subterrânea. Para cada preço, a quantidade demandada é a soma das quantidades demandadas de águas superficial e subterrânea pelos setores na bacia. $\mathrm{O}$ equilíbrio ocorre no ponto $A$, onde a curva de demanda agregada $(D)$ cruza a curva de custo marginal $(C M)$.

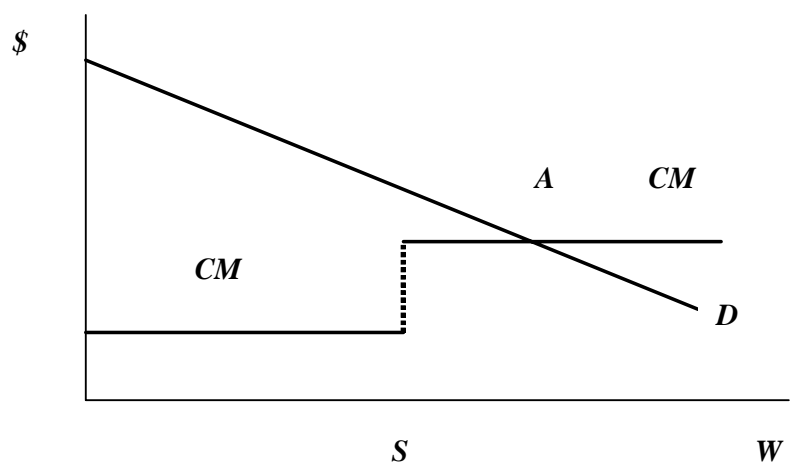

Figura 2 - Mercado competitivo de água (superficial e, ou, subterrânea).

Se $D(\cdot)$ for considerada uma razoável aproximação da curva de demanda compensada da renda, então o benefício social associado à captação de água $W(t)$ é a área sob a curva de demanda de zero a $W(t)$ (ver, por exemplo, CONRAD e CLARK, 1987, p.72). O benefício social bruto pode ser calculado de acordo com a seguinte expressão:

$$
B\left(W_{t}\right)=\int_{0}^{S_{t}+G_{t}} D(W) d W .
$$

Considerando o custo marginal de captação de água superficial, $C$, o custo marginal de captação de água subterrânea, $K$, o custo marginal externo, $E$, e as taxas por volume captado de água superficial $\left(F_{S}\right)$ e água subterrânea $\left(F_{G}\right)$ na bacia, o benefício social líquido da captação 
de água pode ser calculado da seguinte forma: $N B(t)=B(W(t))-C S(t)-E \delta S(t)-F_{S} S(t)-K G(t)-F_{G} G(t) \quad$, em que $0<\delta<1$ é um parâmetro que mede a proporção de água superficial captada na bacia e que retorna aos cursos de água contendo poluentes.

Tendo em vista que se assumiu que a reposição de águas superficial e subterrânea depende do estoque total de água na bacia no ano $t, Z(t)$, os estoques de águas superficial e subterrânea comportam-se de acordo com as seguintes equações diferenciais:

$$
\begin{aligned}
& d X(t) / d t=\dot{X}(t)=\alpha Z(t)-S(t), X(0)=X_{0} \\
& d Y(t) / d t=\dot{Y}(t)=\beta Z(t)-G(t), Y(0)=Y_{0}
\end{aligned}
$$

em que $X(t)$ e $Y(t)$ representam os estoques de água superficial e água subterrânea no período $t$, respectivamente, com $Z(t)=X(t)+Y(t)$; $0<\alpha<1$ e $0<\beta<1$ são parâmetros que representam proporções do estoque total de água na bacia, reposta como água superficial e água subterrânea, respectivamente.

O problema do gestor de recursos hídricos consiste em escolher trajetórias ótimas de captação de água superficial e água subterrânea, sujeita às restrições (1) e (2) e que maximize o valor presente total dos benefícios sociais líquidos no intervalo de tempo $[0, T]$. Formalmente, o problema do gestor de recursos hídricos consiste em (considerou-se, inicialmente, que $\left.F_{S}=F_{G}=0\right):{ }^{6}$

6 Ver, por exemplo, Kamien e Schwartz (1991) para detalhes sobre a formulação e obtenção das condições de primeira e segunda-ordem de otimização de problemas de controle ótimo. 


$$
\underset{\{S(t), G(t)\}}{\operatorname{Max}} \int_{0}^{T}[B(W(t))-C S(t)-E \delta S(t)-K G(t)] e^{-r t} d t,
$$

sujeito às equações (1) e (2) e às restrições de não-negatividade dos estoques e captações de água $S(t), G(t), X(t)$ e $Y(t)$; $r$ é a taxa de desconto, considerada positiva e constante. Neste problema, $X(t)$ e $Y(t)$ são as variáveis de estado; suas derivadas são as equações de transição; e as funções $S(t)$ e $G(t)$ são as variáveis de controle. Nessa formulação do problema de controle ótimo, o gestor de recursos hídricos procura um conjunto de funções $(X(t), Y(t), S(t), G(t))$, que maximiza o somatório dado pela expressão (3).

Para resolver o problema (3), formou-se o seguinte Hamiltoniano de valor corrente (a variável $t$ foi suprimida):

$$
H=B(G+S)-C S-E \delta S-K G+\lambda[\alpha Z-S]+\mu[\beta Z-G],
$$

em que $\lambda$ e $\mu$ são as variáveis de co-estado.

Considerando que todas as funções definidas sejam contínuas e diferenciáveis e assumindo uma solução interior, as condições necessárias para maximização são:

$$
\begin{aligned}
& \partial H / \partial S=\partial B(\cdot) / \partial S-C-E \delta-\lambda=0 ; \\
& \partial H / \partial G=\partial B(\cdot) / \partial G-K-\mu=0 ; \\
& \partial H / \partial X=\alpha \lambda+\beta \mu=r \lambda-\dot{\lambda} ; \\
& \partial H / \partial Y=\alpha \lambda+\beta \mu=r \mu-\dot{\mu} ;
\end{aligned}
$$


$\partial H / \partial \lambda=\dot{X}=\alpha Z-S ; \mathrm{e}$

$$
\partial H / \partial \mu=\dot{Y}=\beta Z-G
$$

junto com as condições iniciais $X(0)=X_{0}$ e $Y(0)=Y_{0}$. Nota-se que $\partial B(\cdot) / S_{t}=\partial B(\cdot) / \partial G_{t}=D(\cdot)$. Adicionando as expressões (4) e (5), diferenciando o resultado com relação ao tempo, e levando em conta as expressões (6) e (7), a seguinte equação diferencial pode ser obtida:

$$
2(\partial D(\cdot) / \partial W) \dot{W}=(r-2 \alpha)[D(W)-C-\delta E]+(r-2 \beta)[D(W)-K] \cdot
$$

Adicionando também as expressões (1) e (2), o resultado é:

$$
\dot{Z}=(\alpha+\beta) Z-W
$$

As equações (8) e (9) formam um sistema de equações diferenciais que devem ser satisfeitas simultaneamente. Em equilíbrio, isto é, quando $\dot{W}=0$ e $\dot{Z}=0$, a solução deste sistema deve satisfazer às seguintes equações (curvas):

$$
\begin{aligned}
& D(W)=[(r-2 \alpha)(C+E \delta)+(r-2 \beta) K] /[2 r-2(\alpha+\beta)], \\
& W=(\alpha+\beta) Z
\end{aligned}
$$

Essas curvas são comumente conhecidas como W-isoclina e Z-isoclina, respectivamente. Qualquer interseção dessas duas curvas é um ponto de equilíbrio do sistema de equações diferenciais. Se a solução satisfizer às condições necessárias do problema de controle ótimo, esse equilíbrio será único e poderá ser representado por $\left(W^{*}, Z^{*}\right)$. Esse equilíbrio será 
estável, se $\lim _{t \rightarrow \infty} W(t)=W^{\bullet}$ e $\lim _{t \rightarrow \infty} Z(t)=Z^{*}$. Se o estoque inicial de água estiver acima do equilíbrio, os usuários captarão esse recurso até que o estoque de equilíbrio seja reestabelecido; se estiver abaixo do equilíbrio, nada será captado até que o equilíbrio seja reestabelecido. Se os usuários agirem dessa forma, o uso do recurso tornar-se-á sustentável, e a água poderá ser explorada indefinidamente.

Considere-se, agora, o comportamento do preço da água sem a cobrança pelo seu uso. Em um mercado competitivo, o equilíbrio ocorrerá quando o preço da água igualar-se ao seu custo marginal de extração. Por um lado, se não houver custos externos e se os custos marginais de extração tanto de água superficial quanto de água subterrânea forem iguais, então $P=C=K$. Por outro, se $K>C$ e não houver custos externos, o preço de mercado da água será igual ao custo de captação de água subterrânea $(P=K)$. Na presença de custos externos, o preço da água será igual ao maior dos seguintes valores: $C+E \delta$ ou $K$. Se o gestor de recursos hídricos cobrar pela água, os custos para o usuário crescerão e, como conseqüência, haverá redução no consumo.

\section{Modelo analítico}

A abordagem de dinâmica de sistemas foi utilizada neste trabalho como modelo analítico na análise da gestão de recursos hídricos em bacias hidrográficas. Essa abordagem baseia-se em mecanismos de retroalimentação (feedback) e simulação orientada a objeto. Ela tem sido utilizada, com relativo sucesso, quando o foco da investigação está na análise e na solução de problemas em sistemas complexos, em que a ênfase está na aprendizagem sobre esses sistemas, ao invés de obter apenas soluções parciais. A esse respeito, ela complementa outros tipos de abordagens, tais como as técnicas de otimização tradicionais. 
A abordagem de dinâmica de sistemas tem sido aplicada para investigar processos complexos em diversas áreas, incluindo, por exemplo, biologia, geografia, estudos ambientais, matemática, psicologia e planejamento de recursos humanos. Recentes aplicações dessa abordagem na área dos recursos hídricos incluem, por exemplo, planejamento do uso de recursos hídricos (SIMONOVIC et al., 1997) e análise da sustentabilidade no uso de recursos hídricos (XU et al., 2002).

Na modelagem de dinâmica de sistemas, devem-se considerar as interações complexas que ocorrem em sistemas. Nesse sentido, a palavra "sistema" deve ser entendida como uma coleção de objetos (entidades ou elementos) que operam de maneira conjunta. Como exemplo, podemse considerar os vários componentes de órgãos, ossos, fluidos e músculos que formam um sistema complexo, o corpo humano. Esses sistemas podem variar em complexidade e tamanho, mas todos eles, de acordo com Boucher (1995, p.4), "têm algumas características em comum e podem ser analisadas usando um conjunto simples de ferramentas".

A base da abordagem de dinâmica de sistemas é o pensamento sistêmico, e uma característica-chave desse pensamento é a interdependência, isto é, a forma como os componentes (elementos) de um sistema estão ligados um ao outro (BRAGA et al., 2004). Essas ligações são descritas por meio de diagramas de influência, que permitem visualizar as interações dos vários componentes de um problema (variáveis, constantes, etc.), suas estruturas de retroalimentação e as defasagens em resposta a mudanças em seus componentes.

Na elaboração de diagramas de influência, podem ser usadas setas para indicar a direção de causalidade entre variáveis, enquanto os sinais (+/-) indicam se o efeito está no mesmo sentido (sinal positivo) ou em sentido oposto (sinal negativo). Em outras palavras, o sinal positivo mostra relação de causalidade direta entre as variáveis, ou seja, um aumento (diminuição) em uma variável causa aumento (diminuição) na outra, enquanto o sinal negativo mostra relação oposta, ou seja, aumento (diminuição) em uma variável causa diminuição (aumento) na outra. Na Figura 3 utiliza-se um 
diagrama de influência para mostrar os ganhos de uma caderneta de poupança que estão associados a determinada taxa de juros.

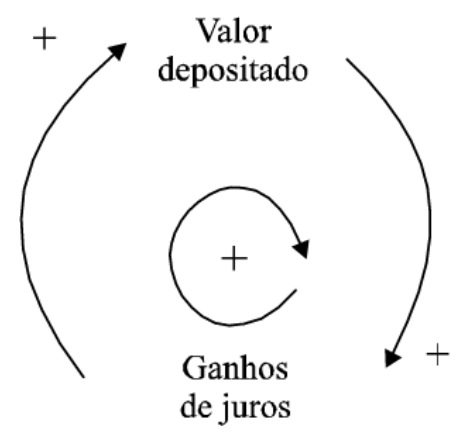

Figura 3 - Diagrama de influência ou causalidade circular.

No caso da Figura 3, a causalidade mostrada é apenas em um sentido (positivo), daí, o sinal “+” indicado nas duas setas mostradas. O processo mostra o acúmulo do volume da poupança com o fluxo contínuo de ganhos de juros ao longo do tempo. O diagrama pode ser interpretado da seguinte forma: "à medida que aumenta o valor depositado na conta poupança, aumenta o montante de ganhos de juros, que, por sua vez, aumenta o valor depositado na poupança, que, na seqüência, aumenta os ganhos de juros, e assim sucessivamente".

Embora os diagramas de influência sejam úteis para descrever sistemas complexos, eles são muito limitados em uma série de aspectos. É o caso, por exemplo, quando se deseja quantificar os efeitos da mudança em uma variável de um sistema sobre as demais ou avaliar cenários alternativos relativos ao mesmo sistema. Entretanto, os diagramas de influência podem constituir um primeiro passo na formalização de problemas de dinâmica de sistemas, que, por meio do uso de computadores, tornam possível a representação explícita de sistemas complexos em forma matematizada, permitindo análises quantitativas e a comparação de cenários alternativos representativos de diferentes políticas (BRAGA et al., 2004). 
Dois componentes principais constituem a estrutura básica de uma estrutura ou modelo de dinâmica de sistemas: estoques e fluxos. Esses componentes são organizados na forma de causa e efeito e, dependendo da natureza do problema, podem incorporar efeitos defasados (delays) no tempo. Os estoques representam acúmulos no sistema, os quais resultam da diferença entre fluxos de entrada e fluxos de saída e retratam o estado do sistema em dado ponto no tempo. No caso dos recursos hídricos, os estoques representam os acúmulos de água na superfície (rios, lagos, etc.) e no subsolo (aqüíferos, etc.). Os fluxos resultam de decisões ou políticas sobre um sistema e afetam os estoques. No caso dos recursos hídricos, são representados pelas taxas de captação de água superficial e, ou, água subterrânea.

Quando um estoque é influenciado por seu estado no passado, tem-se uma situação de retroalimentação (feedback). De forma similar ao de um diagrama de influência, ter-se-á retroalimentação positiva quando o aumento de uma variável causar aumento futuro na mesma variável, ou retroalimentação negativa quando o aumento de uma variável levar a um decréscimo futuro desta. Essa interação dos efeitos de retroalimentação positivos e negativos leva, segundo Braga et al. (2004), à estabilização dos sistemas, que pode resultar em comportamentos assintóticos ou oscilatórios. Os resultados dessas interações podem ser também nãolineares, o que torna difícil a previsão dos efeitos de mudanças em determinadas variáveis sobre o comportamento do sistema.

Do ponto de vista formal, a abordagem de dinâmica de sistemas fundamenta-se na teoria do controle ótimo e na moderna teoria de dinâmica não-linear, ou seja, a teoria e os modelos desenvolvidos que utilizam essa abordagem têm uma fundamentação matemática rigorosa (STERMAN, 2000). Por exemplo, a correspondente representação da função objetivo do problema de controle ótimo e das equações diferenciais da evolução dos estoques de água, apresentados na terceira seção deste trabalho, tomaria, na linguagem de estoques e fluxos da dinâmica de sistemas, o seguinte aspecto (considerando a forma agregada do modelo teórico): 
$\operatorname{Estoque}(t)=\int_{t_{0}}^{t}[$ Fluxo de Entrada $(s)$-Fluxo de Saída $(s)] d s+\operatorname{Estoque}\left(t_{0}\right)$, em que o Fluxo de Entrada(s) representa o valor do fluxo de entrada em qualquer tempo $s$, entre o tempo inicial $t_{0}$ e o tempo corrente $t$. Por sua vez, a taxa líquida de mudança de qualquer estoque, sua derivada, é o fluxo de entrada menos o fluxo de saída, definido pela seguinte equação diferencial:

$d($ Estoque $) / d t=$ Fluxo de Entrada(t)-Fluxo de Saída(t).

Do ponto de vista prático, a modelagem e a simulação de problemas com dinâmica de sistemas em computadores podem ser feitas de duas formas alternativas: a) Programação direta do modelo de simulação mediante uso de alguma linguagem de programação, tais como Fortran, Java, C++, C\#, etc.; ou b) Utilização de pacotes computacionais já prontos, disponíveis no mercado, os quais têm sido cada vez mais utilizados pelos usuários de dinâmica de sistemas. A grande vantagem da segunda alternativa é a possibilidade de o usuário poder concentrar-se apenas no problema a ser modelado, sem ter que se dedicar também à aprendizagem de uma linguagem de programação e ao desenvolvimento, implementação e teste de sistemas próprios para simular modelos de dinâmica de sistemas em computadores.

Existem diversos pacotes computacionais disponíveis no mercado para simulação dinâmica, destacando-se o Powersim, Stella, iThink e Vensim. Segundo Braga et al. (2004), os três primeiros softwares utilizam a mesma forma de representação visual para os diferentes componentes que usualmente compõem um modelo de simulação dinâmica, o que, de certa forma, padroniza os símbolos (ícones) utilizados na representação dos modelos de dinâmica de sistemas. Na Figura 4 mostram-se os principais ícones utilizados na modelagem de dinâmica de sistemas do Stella, software utilizado neste trabalho. 


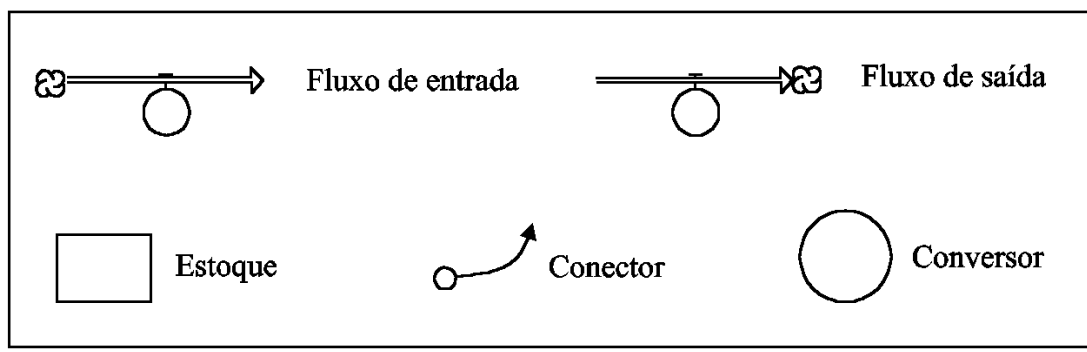

Figura 4 - Ícones do software Stella utilizados na modelagem de dinâmica de sistemas.

Fonte: HPS (2001).

Os estoques e fluxos foram definidos anteriormente. Os estoques são representados por um retângulo e os fluxos, por uma seta cheia (tubo), por um balão (válvula), que indica quantidade por unidade de tempo entre estoques, e por uma "nuvem". Quando não estão vinculadas a estoques, os fluxos possuem uma nuvem na extremidade que indica comunicação com o exterior. A nuvem representa uma fonte inesgotável de um recurso ou uma vazão inesgotável para um estoque. Os círculos são conversores que contêm as relações algébricas do modelo, e os conectores são utilizados para estabelecer links de informações dentro do modelo.

Para ilustrar como os ícones apresentados acima podem ser utilizados para modelar um sistema dinâmico, considere o diagrama de influência apresentado na Figura 3, de cômputo de juros compostos. O estoque representa o valor acumulado em depósitos e os fluxos, os ganhos por unidade de tempo, de acordo com o valor depositado e a taxa de juros. Quanto maior for o valor depositado, dada a taxa de juros, maior será o fluxo de ganhos. Para indicar que o valor do depósito influencia os fluxos de ganhos, utiliza-se um conector que liga o estoque ao ícone do fluxo. Por fim, utiliza-se um conversor, ligado por um conector ao ícone de fluxo, para calcular os fluxos de ganhos, dada a taxa de juros e dado o volume de depósito. A modelagem desse problema de juros compostos, com dinâmica de sistemas, é mostrada na Figura 5. 


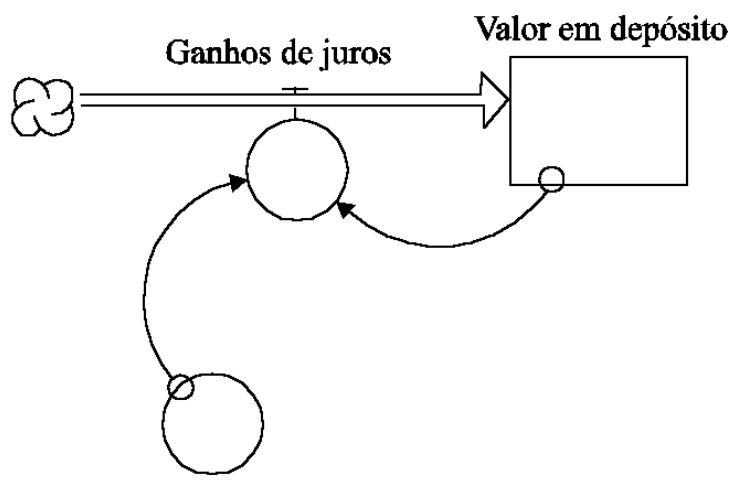

Taxa de juros

Figura 5 - Modelagem com dinâmica de sistemas do cálculo de juros compostos.

Para tornar o modelo de dinâmica de sistemas apresentado na Figura 5 operacional, devem-se nomear seus vários ícones, atribuir valores às variáveis ou parâmetros e estabelecer as relações algébricas entre estoques e fluxos. Na Figura 5, o estoque foi nomeado de "Valor em Depósito"; o fluxo, de "Fluxos de Ganhos"; e o conversor, de "Taxa de Juros". Ao clicar nesses nomes, podem-se atribuir valores (utilizaram-se $\mathrm{R} \$ 100,00$ como depósito inicial e taxa de juros igual a 0,06 ) ou estabelecer relações algébricas, conforme indicado a seguir:

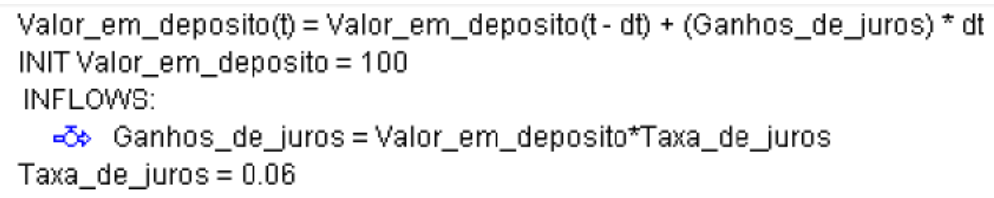

Caso o modelo esteja completo, ou seja, os ícones estejam ligados de forma consistente na modelagem de dado problema; caso cada ícone esteja nomeado; e caso tenham sido atribuídos valores às variáveis e aos 
parâmetros e estabelecidas as relações algébricas, este estará pronto para ser "rodado". Os resultados do modelo são usualmente apresentados na forma de gráficos, que mostram a evolução, no tempo, das variáveis de interesse ou séries numéricas que podem ser organizadas em tabelas. Com base nos resultados, valida-se o modelo, ou seja, verifica-se se este é consistente com a realidade modelada ou não. Após validado, o modelo estará pronto para realização de análises de sensibilidade (simulações), mediante mudanças nos valores iniciais dos parâmetros. No caso do cômputo dos juros compostos, por exemplo, uma análise de sensibilidade poderia ser realizada mediante variação na taxa de juros.

O exemplo utilizado para exemplificar a modelagem de dinâmica de sistemas, cômputo de juros compostos, é extremamente simples. Entretanto, a abordagem de dinâmica de sistemas pode ser utilizada para modelar problemas com elevado grau de complexidade de forma relativamente simples, e isso pode ser facilmente constatado ao consultar o manual do usuário do software Stella (HPS, 2001), por exemplo, ou autores da área, como Sterman (2000), para problemas diversos, ou Ruth e Hannon (1997), para problemas na área de economia. Entretanto, a modelagem de problemas simples pode constituir um passo importante para ganhar confiança para os desafios que surgem com os problemas mais complexos. Pode ser útil também estabelecer certa rotina na modelagem de problemas com dinâmica de sistemas.

Ford (1999), por exemplo, sugere oito passos para a modelagem com dinâmica de sistemas: a) Familiarizar-se com o problema a ser modelado e com os elementos nele envolvidos; b) Desenhar os diagramas de influência; c) Estabelecer os estoques, fluxos e variáveis auxiliares do problema; d) Definir os valores para os parâmetros do modelo e estabelecer as relações algébricas entre estoques e fluxos; e) Rodar o modelo e comparar o resultado obtido com a realidade; se o resultado for incompatível, rever o processo de modelagem a partir do passo dois; f) Realizar análises de sensibilidade; e g) Avaliar os efeitos de novas políticas, se for o caso. 


\section{Aplicação do modelo à bacia do rio Araguari}

O modelo desenvolvido na terceira seção deste trabalho permite considerar diversas situações. Permite, por exemplo, dividir uma bacia hidrográfica em várias regiões, em que cada região acomodará mais de um setor que demanda água, e discriminar fontes de água e, ou, setores. Por sua vez, diferentes critérios podem ser usados para definir o montante a ser pago pelos usuários de água em uma bacia hidrográfica. $\mathrm{O}$ gestor de recursos hídricos poderá não utilizar nenhum tipo de discriminação ou querer discriminar fontes de água (superficial e subterrânea) entre regiões em uma bacia hidrográfica ou entre setores (urbano, industrial e agrícola, por exemplo), ou simplesmente cobrar pela água, tendo em vista determinado volume de recursos a ser arrecadado.

Embora o modelo proposto possa ser utilizado para implementar essas várias possibilidades de cobrança pelo uso de água, foram consideradas apenas duas para ilustrar sua aplicação: não-discriminação e discriminação entre fontes de água e entre setores usuários desse recurso. Assim, na aplicação do modelo à bacia do rio Araguari, foram consideradas as seguintes situações: a) Apenas uma região e um setor com e sem discriminação entre fontes de água (superficial e subterrânea) e cinco anos de horizonte de planejamento; ${ }^{7}$ b) Duas regiões, cada uma com três setores (urbano, industrial e agrícola) e cinco anos de horizonte de planejamento. ${ }^{8}$ Nota-se que, nessas duas situações de aplicação do modelo, foram ignorados os custos externos e os custos de distribuição de água.

Nota-se também que, no caso de discriminação (entre fontes de água e, ou, entre setores), as curvas de demanda foram consideradas separadamente na aplicação do modelo. Outras simplificações adotadas

\footnotetext{
Embora certas outorgas em Minas Gerais tenham validade por até 20 anos, as primeiras experiências de cobrança pelo uso de água em bacias hidrográficas no Brasil têm mostrado a necessidade de revisão das taxas/tarifas, após certo período de tempo. Ver, por exemplo, Formiga-Johnsson et al. (2007), no caso da bacia do rio Paraíba do Sul. Em razão disso, considerou-se, como horizonte de planejamento, um período de cinco anos, que é também o período de validade da maioria das outorgas em Minas Gerais.

8 A demanda de água residencial está sendo usada como "proxy" para a demanda das agências que fornecem serviços de água encanada e tratada para uso doméstico.
} 
na implementação do modelo foram: a) Os custos marginais de captação de águas superficial e subterrânea foram considerados iguais para todas as regiões e todos os setores; $;^{9}$ b) As curvas de demandas foram consideradas lineares e as elasticidades-preços foram obtidas de estimações, feitas por outros autores, para o Brasil e para outros países, ${ }^{10}$ e c) A alocação de água na bacia do rio Araguari atingiu seu estado estacionário.

Na primeira simulação com o modelo, foram considerados os seguintes valores para os parâmetros: a) Custo marginal de captação de água (superficial ou subterrânea) igual a 225,00 ( $C=K=225,00$ ); b) Elasticidades-preços das demandas agregadas de água iguais a $\varepsilon=-0,7$ e $\varepsilon=-0,9$, no caso de não-discriminação entre fontes de água, e igual a $\varepsilon=-0,8$, no caso de discriminação entre fontes de água; c) Taxa de desconto igual a 0,06 ; d) Taxas iguais a $\mathrm{R} \$ 20,00$ por mil $\mathrm{m}^{3}$ de água captada, no caso de não-discriminação entre fontes de água, e R \$20,00 por mil $\mathrm{m}^{3}$ de água subterrânea e $\mathrm{R} \$ 5,00$ por $\mathrm{mil}^{3}$ de água superficial, no caso de discriminação entre fontes de água; ${ }^{11}$ e) Condições iniciais de estoques, $X_{0}=\alpha Z=1.705 .927,88$ e $Y_{0}=\beta Z=87.690,84$, na versão com discriminação entre fontes de água, e $Z_{0}=1.793 .618,72$, na versão sem discriminação entre fontes de água.

O objetivo desta primeira simulação foi verificar o comportamento das receitas provenientes da cobrança pelo uso da água, ao longo de um período de cinco anos e a redução no consumo de água no mesmo período,

\footnotetext{
Utilizou-se, como custo marginal de captação, o custo de energia elétrica para captar mil m3 de água. A energia elétrica é o principal insumo variável relacionado com captação de água na bacia do rio Araguari.

${ }^{10}$ Bhatia et al. (1995) estimaram em -0,6 a elasticidade-preço da demanda residencial de água no Brasil e em -1,32 a elasticidade-preço da demanda industrial de água na Índia. A demanda de água para irrigação sofre, em geral, influência de diversos fatores (regional, estrutural, condições institucionais e fatores sazonais) e, dependendo da situação, pode ser elástica ou inelástica (ver, por exemplo, VARELA-ORTEGA et al., 1998; e WHEELER et al., 2008).

11 Em 2006, foi estabelecido um preço unitário (m3) de água captada na bacia do rio Paraíba do Sul, região sudeste do Brasil, de $\mathrm{R}$ \$ 0,02 para usos industrial, municipal e mineração, e um preço unitário de $\mathrm{R} \$ 0,0005$ para uso agrícola (FORMIGA-JOHNSSON et al., 2007).
} 
em resposta a mudanças na elasticidade-preço da demanda agregada de água e discriminação entre fontes de água na bacia do rio Araguari. Os pares preços-quantidades $\left(C, Z_{0}\right)$, no caso de não-discriminação, e $(C$, $\left.X_{0}\right)$ e $\left(K, Y_{0}\right)$, no caso de discriminação, foram considerados os equilíbrios iniciais, e os interceptos das curvas de demandas foram calculados com base nos equilíbrios iniciais e nas elasticidades-preços. Os resultados dessa primeira simulação são apresentados na Tabela 2, e o modelo de dinâmica de da parte 'sem discriminação' encontra-se no Apêndice (Figuras 1A e 2A).

Tabela 2 - Receitas da cobrança pelo uso da água de acordo com diferentes elasticidades-preços da demanda e discriminação entre fontes de água, bacia do rio Araguari, Minas Gerais

\begin{tabular}{lrrrr}
\hline \multirow{2}{*}{ Ano } & \multicolumn{2}{c}{ Sem discriminação } & \multicolumn{2}{c}{ Com discriminação } \\
\cline { 2 - 5 } & \multicolumn{1}{c}{$\varepsilon=-0,7$} & \multicolumn{1}{c}{$\varepsilon=-0,9$} & Água superficial & \multicolumn{1}{c}{ Água subterrânea } \\
\hline 1 & $33.640 .301,40$ & $33.002 .608,40$ & $8.378 .002,95$ & $1.629 .099,20$ \\
2 & $31.621 .883,32$ & $31.022 .451,90$ & $7.875 .322,77$ & $1.531 .353,25$ \\
3 & $29.724 .570,00$ & $29.161 .104,78$ & $7.402 .803,41$ & $1.439 .472,05$ \\
4 & $27.941 .096,10$ & $27.411 .438,50$ & $6.958 .635,20$ & $1.353 .103,73$ \\
5 & $26.264 .630,33$ & $25.766 .752,19$ & $6.541 .117,09$ & $1.271 .917,51$ \\
\hline Total & $149.192 .481,46$ & $146.364 .355,76$ & $37.155 .881,42$ & $7.244 .945,74$ \\
\hline
\end{tabular}

Fonte: Resultados da pesquisa.

Os resultados da Tabela 2 mostram que as receitas provenientes da cobrança pelo uso de água na bacia do rio Araguari não diferem significativamente em resposta a diferentes elasticiadades-preços da demanda agregada de água e não-discriminação entre fontes de água. Por um lado, no período de cinco anos, o valor presente total das receitas será igual a R\$ 149.192.481,46, se a elasticidade-preço da demanda for -0,7, e será igual a $\mathrm{R} \$ 146.364 .355,76$, se a elasticidade-preço da demanda for igual a -0,9. Por outro, se for discriminada a fonte de água, as receitas poderão variar significativamente, especialmente se for cobrada uma baixa taxa para a fonte de água mais abundante, que, no caso da bacia do rio Araguari, é a água superficial. As reduções anuais no consumo de água na bacia, com cobrança pela água (não mostradas na Tabela 2), 
variam de 111.600 a $143.488 \mathrm{mil} \mathrm{m}^{3}$, no caso de não-discriminação, e de $30.327 \mathrm{mil} \mathrm{m}^{3}$ de água superficial a $6.235 \mathrm{mil} \mathrm{m}^{3}$ de água subterrânea, no caso de discriminação entre fontes de água. Nota-se que esses valores mudarão se diferentes valores para o custo marginal de captação de água forem utilizados.

Na próxima simulação, os seguintes valores de parâmetros foram utilizados: a) Custo marginal de captação de água, $C=K=225,00$; b) Taxa de desconto, 0,06; c) Elasticidade-preço da demanda urbana de água, $\varepsilon=-0,6$; d) Elasticidade-preço da demanda de água para irrigação, $\varepsilon=-0,8$; e) Elasticidade-preço da demanda de água para uso industrial, $\varepsilon=-1,2$; f) Taxas de $\mathrm{R} \$ 20,00$ por mil $\mathrm{m}^{3}$ de água captada para uso urbano e industrial e de $\mathrm{R} \$ 2,00$ por mil $\mathrm{m}^{3}$ de água para irrigação; e g) Condições iniciais de estoques obtidos da Tabela 1. O objetivo dessa simulação foi verificar o comportamento das receitas da cobrança pelo uso de água e das reduções no consumo em resposta a diferentes elasticidades-preços das demandas de água setoriais e discriminação entre setores nas duas regiões da bacia do rio Araguari. Os resultados obtidos estão apresentados na Tabela 3 e, no Apêndice, encontra-se o sub-modelo de dinâmica de sistemas do setor urbano, região I (Figuras $3 \mathrm{~A}$ e $4 \mathrm{~A}){ }^{12}$

Tabela 3 - Receitas obtidas da cobrança pelo uso de água com discriminação entre setores nas regiões I e II, bacia do rio Araguari, Minas Gerais

\begin{tabular}{lrllrcr}
\hline Ano & \multicolumn{3}{c}{ Região I } & & \multicolumn{3}{c}{ Região II } \\
\hline & Urbano & Agrícola & Industrial & Urbano & Agrícola & \multicolumn{1}{c}{ Industrial } \\
\hline 1 & $2.894 .492,40$ & $414.843,48$ & $879.945,00$ & $439.861,00$ & $2.693 .289,54$ & $54.760,80$ \\
2 & $2.720 .822,86$ & $389.952,87$ & $827.148,30$ & $413.469,34$ & $2.531 .692,17$ & $51.475,15$ \\
3 & 2.557 .573 .48 & $366.555,70$ & $777.519,40$ & $388.661,18$ & $2.379 .790,64$ & $48.386,64$ \\
4 & $2.404 .119,08$ & $344.562,36$ & $730.868,24$ & $365.341,51$ & $2.237 .003,20$ & $45.483,44$ \\
5 & $2.259 .871,93$ & $323.888,62$ & $687.016,14$ & $343.421,02$ & $2.102 .783,01$ & $42.754,44$ \\
\hline Total & $12.836 .879,75$ & $1.839 .803,02$ & $3.902 .497,08$ & $1.950 .754,05$ & $11.944 .558,55$ & $242.860,48$ \\
\hline
\end{tabular}

Fonte: Resultados da pesquisa.

${ }^{12}$ Os demais sub-modelos dos outros setores e regiões são similares. 
Como pode ser observado na Tabela 3 , as receitas provenientes da cobrança pelo uso de água na bacia do rio Araguari variam de acordo com o setor e com a região considerados. Na mesma região, as receitas variam também de acordo com a importância relativa do setor e com a taxa cobrada pelo uso da água. Na região I, a principal fonte de receita vem do setor urbano e, na região II, do setor agrícola, mesmo considerando, nesse último caso, uma baixa taxa para uso da água ( $\mathrm{R} \$$ 2,00 por mil $\mathrm{m}^{3}$ de água captada). As reduções no consumo de água (não mostradas na Tabela 3), com a introdução da cobrança pelo seu uso (em mil $\mathrm{m}^{3}$ ), foram: a) Região I: urbano $(8.152,73)$, agrícola $(1.485,37) \mathrm{e}$ industrial $(5.253,38)$; e b) Região II: urbano $(1.237,16)$, agrícola $(9.644,40)$ e industrial $(326,20)$.

Na última simulação, os estoques de água disponíveis para cada setor e cada região foram considerados conhecidos a priori. De fato, foram utilizados os valores das outorgas de água, válidas em $2006\left(\mathrm{mil} \mathrm{m}^{3}\right)$, disponibilizadas pelo IGAM em seu site. Todavia, o modelo pode identificar a melhor alocação de água entre setores em dada região. As informações básicas necessárias incluem a demanda de água de cada setor, em cada região, e os estoques de água (superficial e subterrânea) disponíveis para consumo em cada região. As demandas setoriais de água devem ser estimadas e os estoques de água disponíveis para consumo em cada região como um todo, podem ser definidos pela agência responsável pela concessão de outorgas.

Embora tenham sido utilizadas curvas de demanda de água não especificamente estimadas para a bacia do rio Araguari, os valores simulados permitem estimar o volume a ser arrecadado e verificar sua compatibilidade com o plano de recursos hídricos aprovado para a bacia. Se for o caso, os critérios de cobrança poderão ser alterados e os valores a serem cobradas poderão ser ajustados. Dado que há perdas de bemestar dos usuários, com a cobrança pelo uso de água, é conveniente que os recursos arrecadados sejam utilizados prioritariamente na própria bacia. Com isso, haveria, certamente, maior predisposição dos usuários de água 
em pagar as taxas acordadas no processo de negociação, no âmbito dos comitês de bacia.

Outro aspecto a ser destacado diz respeito à abordagem de dinâmica de sistemas. Dado que o sistema de outorgas é, de certa forma, controlado exogenamente no Estado de Minas Gerais pelo IGAM, isso deixou pouca margem para explorar mais recursos desse tipo de abordagem, especialmente os aspectos dinâmicos de ajustamentos, tais como realocações de água entre setores e entre regiões na bacia. Outras situações de ajustamentos poderiam também ter sido simuladas, tais como aumento na demanda de água devido ao crescimento populacional e, ou, aumento na atividade econômica na região, mais isso exigiria estimativas confiáveis de crescimento dessas variáveis para se obterem resultados consistentes.

\section{Considerações finais}

A água é um recurso tão complexo quanto importante, razão pela qual é crucial o desenvolvimento de instituições econômicas e políticas para sua distribuição. Do ponto de vista econômico, os mercados competitivos possuem propriedades desejáveis que os tornam o melhor benchmark na definição de políticas para a água. Os mercados competitivos podem ser usados como referência, mesmo em situações em que a alocação de água não esteja baseada em mercados, como é o caso do Brasil, onde, de acordo com a legislação, sua distribuição deve passar por um processo de negociação em bases locais (bacias hidrográficas). Em tais situações, o processo de barganha pode levar as negociações a resultados próximos ou iguais aos de mercados competitivos.

Apresentou-se, neste trabalho, um modelo dinâmico para alocar água renovável (água superficial e água subterrânea) entre diferentes usuários, em uma bacia hidrográfica. Com base em sua solução, foram sugeridos critérios para cobrança pelo uso de água e analisou-se a sustentabilidade no uso desse recurso. O modelo baseou-se no princípio fundamental de 
que, em um mercado competitivo, a eficiência econômica será maximizada quando os benefícios marginais (sociais e privados) forem igualados ao preço de mercado da água. O modelo foi aplicado, mediante uso de dinâmica de sistemas, à bacia hidrográfica do rio Araguari, localizada no estado de Minas Gerais.

Para alocar água de forma eficiente em uma bacia hidrográfica, será necessária também uma coordenação entre o comitê da bacia e a agência governamental responsável pelas outorgas. Todas as outorgas em uma bacia hidrográfica devem ser ajustadas ao novo regime de cobrança pelo uso de água, uma vez que haverá redução no consumo pelos usuários. Esse ajustamento implicará a realocação de água entre regiões e entre setores de uma região específica da bacia hidrográfica. Todos esses ajustamentos devem basear-se em informações confiáveis sobre custos de captação de água, custos externos, funções de demandas setoriais e disponibilidade de água em uma bacia hidrográfica.

Finalmente, embora na abordagem de dinâmica de sistemas tenham sido levados em conta os novos arranjos institucionais e as leis brasileiras para gerir os recursos hídricos, ela pode ser facilmente adaptada a outros contextos de uso múltiplo da água não estritamente relacionado com uma bacia hidrográfica. Outros contextos que poderiam ser considerados incluem: a) Considerar apenas uma fonte de água (superficial ou subterrânea); b) Incluir os custos de distribuição de água; c) Considerar outras estruturas de mercado para alocação de água; d) Incluir usos não-consuntivos de água, tais como água para navegação, turismo e, ou, serviços ambientais; e) Detalhar os custos externos associados ao consumo de água; e g) Considerar situações de aumento na demanda de água, em decorrência do crescimento populacional e, ou, do aumento da atividade econômica na região sob análise. 


\section{Referências}

BHATIA, R.; CESTTI, R.; WINPENNY, J. Water conservation and reallocation: best practice cases in improving economic efficiency and environmental quality, World Bank - ODI Joint Study, 1995.

BOUCHER, A. Systems modelling in economics: use of object-oriented software, CHEER, v. 9, n. 2, 1995, p.1-15.

BRAGA, J. L.; SILVA, C. A. B.; WIAZOWSKI, B. A.; AVELLAR, S. O. C. Modelagem com dinâmica de sistemas. In: SANTOS, M. L.; VIEIRA, W. C. Métodos quantitativos em economia. Viçosa: Editora UFV, 2004. p.411-431.

BRILL, E.; HOCHMAN, E.; ZILBERMAN, D. Allocation and pricing at the water district level, American Journal of Agricultural Economics, 79(3):952-963, 1997.

CONRAD, J. M.; CLARK, C. W. Natural resource economics: notes and problems. New York: Cambridge University Press, 1987. 231p.

FORD, A. Modeling the environment: an introduction to systems dynamics modeling of environmental systems. Washington, D.C.: Island Press, 1999. 401p.

FORMIGA-JOHNSSON, R.M.; KUMLER, L.; LEMOS, M. C. The politics of bulk water pricing in Brazil: lessons from the Paraiba do Sul basin, Water Policy, 9:87-104, 2007.

HPS - HIGH PERFORMANCE SYSTEMS, INC. An introduction to systems thinking - STELLA. Hanover, N.H., U.S.A., 2001.

IBGE - INSTITUTO BRASILEIRO DE GEOGRAFIA E ESTATÍSTICA - Contagem da população - 2007. Disponível em: http://www.ibge.gov.br. Acesso em: Fevereiro/2008. 
IGAM - INSTITUTO MINEIRO DE GESTÃO DAS ÁGUAS. Listagem completa de outorgas - 2007. Disponível em: http:// www.igam.mg.gov.br. Acesso em: Fevereiro/2008.

KAMIEN, M.I.; SCHWARTZ, N.L. Dynamic optimization: the calculus of variations and optimal control in economics and management. North-Holland, Amsterdam, 1991.

LEAL, M. S. Gestão ambiental de recursos hídricos: princípios e aplicações. Rio de Janeiro: CPRM, 1998. 176p.

LEGISLAÇÕES FEDERAIS E ESTADUAIS SOBRE RECURSOS HÍDRICOS. Disponível em: http://www.abrh.org.br. Acesso em: Julho/ 2007.

REVISTA SUL AMBIENTAL. Água, recurso finito. Disponível em: http://sulambiental.com.br/edicao_02/agua 02b.htm. Acesso em: Dezembro/2003.

RUTH, M.; HANNON, B. Modeling dynamic economic systems. New York: Springer, 1997.339p.

SIMONOVIC, S.P.; FAHMY, H.; EL-SHORBAGY, A. The use of object-oriented modeling for water resources planning in Egypt, Water Resources Management, 11:243-261, 1997.

STERMAN, J. D. Business dynamics: systems thinking and modeling for a complex world. New York: Irwin McGraw-Hill, 2000. 982p.

TUNDISI, J.G. Ciclo hidrológico e gerenciamento integrado. Ciência e Cultura, São Paulo, v. 55, n. 4, p. 31-33, out./dez. 2003.

VARELA-ORTEGA, C.; SUMPSI, J.M.; GARRIDO, A.; BLANCO, M.; IGLESIAS, E. Water pricing policies, public decision making and farmers' response: implications for water policy, Agricultural Economics, 19: 193-202, 1998. 
WHEELER, S.; BJORNLUND, H.; SHANAHAN, M.; ZUO, A. Price elasticity of water allocations demand in the Goulburn-Murray irrigation district, The Australian Journal of Agricultural and Resource Economics, 52: 37-55, 2008.

XU, Z.X.; TAKEUCHI, K.; ISHIDAIRA, H.; ZHANG, X. W. Sustainability analysis for Yellow River water resources using the system dynamics approach, Water Resources Management, 16:239-261, 2002.

Abstract: The objective of this work was to analyze both the allocation of water among competing demands in a river basin and pricing policies for water. Based on a theoretical model that maximizes the net social benefits from the allocation of water over a finite period of time, it was used the dynamic systems approach to analyze various issues related to water resources management in a river basin, such as sustainability of water resources, and water pricing. The model was applied to the Araguari River Basin, located in the State of Minas Gerais.

Keywords: river basin, system dynamics, water resources, water pricing, sustainability. 


\section{Apêndice}

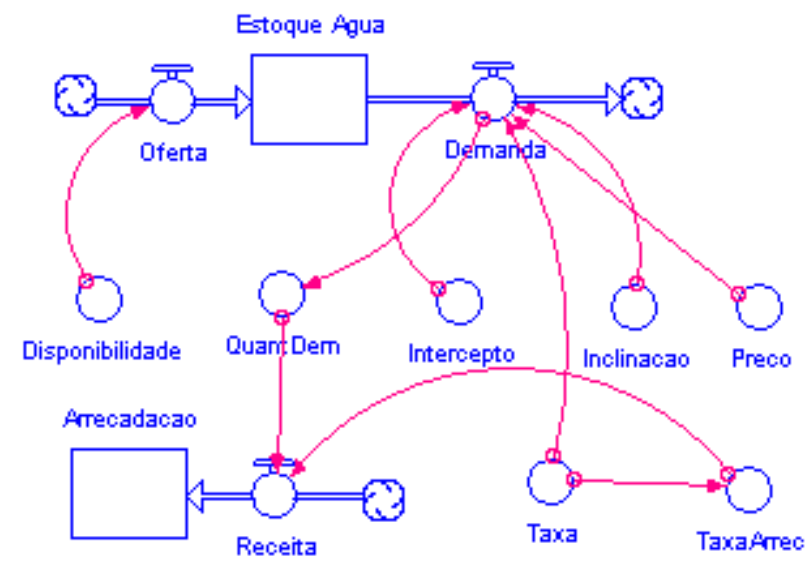

Figura 1A - Modelo de dinâmica de sistemas da bacia do rio Araguari com uma região, um setor e não-discriminação entre fontes de água.

Fonte: Resultados da pesquisa. 
INFLOWS:

$\triangle$ Receita $=$ QuantDem*TaxaArrec

$\square$ Estoque_Agua(t) $=$ Estoque_Agua(t $-d t)+($ Oferta - Demanda $){ }^{*} \mathrm{dt}$

INIT Estoque_Agua = Oferta

INFLOWS:

$\Rightarrow$ Oferta $=$ Disponibilidade

OUTFLOWS:

$\Rightarrow \varnothing$ Demanda $=$ Intercepto-Inclinacao* $($ Preco+Taxa)

Disponibilidade $=1793618.72$

$\bigcirc$ Inclinacao $=5580.15$

$\bigcirc$ Intercepto $=3049151.82$

- Preco $=225$

QuantDem = Demanda

Taxa $=20$

- TaxaArrec $=$ Taxa

Figura 2A - Equações do modelo de dinâmica de sistemas da Figura $1 \mathrm{~A}$. Fonte: Resultados da pesquisa. 


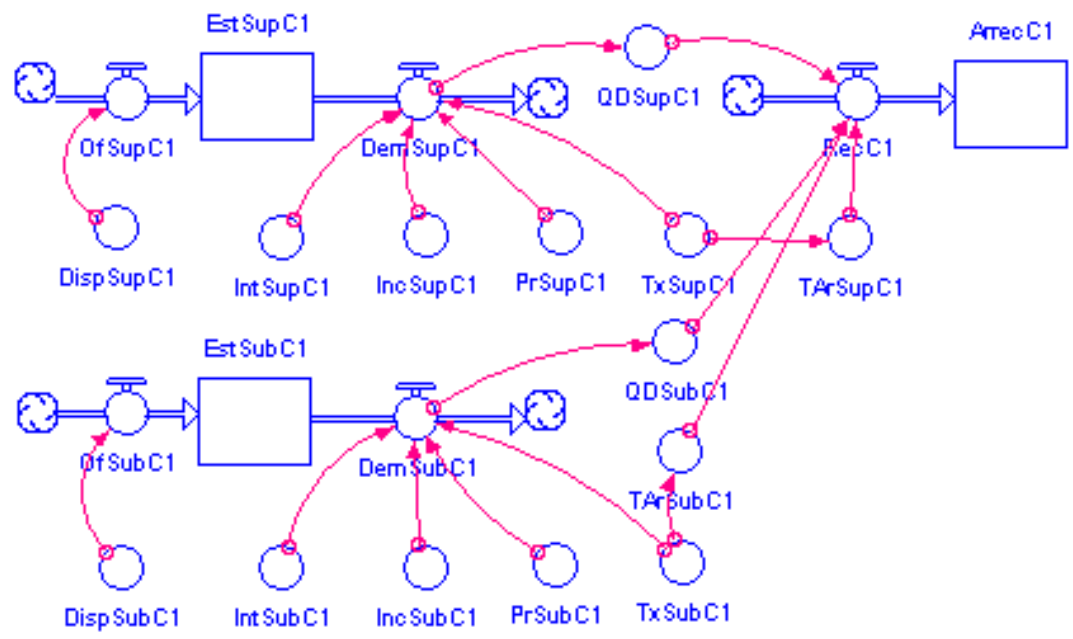

Figura 3A - Modelo de dinâmica de sistemas da bacia do rio Araguari com duas regiões, três setores e discriminação entre setores (sub-modelo setor urbano, região I).

Fonte: Resultados da pesquisa. 
ArrecC1 $(t)=\operatorname{ArrecC} 1(t-d t)+(\operatorname{RecC} 1) * d t$

INIT ArrecC1 $=$ RecC1

INFLOWS:

$\Rightarrow$ RecC1 = QDSupC1*TArSupC1+QDSubC1*TArSubC1

$\square$ EstSubc1 $(t)=E s t S u b c 1(t-d t)+($ OfSubc1 - DemSubc1) * dt

INIT EstSubC1 = OfSubC1

INFLONS:

$\Rightarrow$ OfSubc1 = DispSubc1

OUTFLOWS:

$\Longrightarrow \bar{D}$ DemSubc1 = IntSubc1-IncSubc1*(PrSubC1+TxSubc1)

$\square$ EstSupC1 $(\mathrm{t})=\mathrm{EstSupC1}(\mathrm{t}-\mathrm{dt})+(\mathrm{OfSupC} 1-\mathrm{DemSupC} 1){ }^{*} \mathrm{dt}$

INIT EstSupC1 = OfSupC1

INFLOW':

$\Rightarrow$ OfSupC1 = DispSupC1

OUTFLOWS:

$\triangle \not \Rightarrow$ DemSupc1 = IntSupC1-IncSupc1 (PrSupC1+TxSupC1)

DispSubC1 $=20465.10$

DispSupC1 $=132412.25$

IncSubc1 $=54.57$

$\bigcirc \operatorname{IncSupC} 1=353.10$

IntSubc1 $=32744.17$

IntSupC1 $=211859.60$

PrSubc1 $=225$

$\bigcirc$ PrSupC1 $=225$

QDSubc1 = DemSubc1

QDSupC1 $=$ DemSupC1

TArSubC1 $=$ TxSubC1

TArSupC1 $=$ TxSupC1

TxSubc1 $=20$

TxSupC $1=20$

Figura 4A -Equações do sub-modelo de dinâmica de sistemas da Figura $3 \mathrm{~A}$.

Fonte: Resultados da pesquisa. 